\title{
An updated checklist of the Centipedes (Myriapoda Chilopoda) of Cameroon, with new distribution records
}

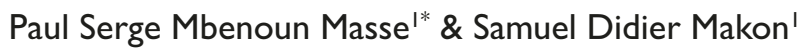 \\ ${ }^{1}$ University of Yaounde I, Faculty of Science, Laboratory of Zoology, PO Box: 812 Yaounde, Cameroon \\ ${ }^{*}$ Corresponding author, email: masseserge@yahoo.fr; smbenoun@uy1.uninet.cm
}

\begin{abstract}
A checklist of Centipedes (Chilopoda) for Cameroon with updated nomenclature of old records and recent additional records from Cameroon's fauna is provided. The provisional list reveals nine endemic species (39\%), four new distribution records, and includes four species that were previously omitted, thereby leading to a modest extension of the list from 19 to 23 species. Several unidentified or doubtful species no longer recognized from the African continent are excluded from the list.
\end{abstract}

KEY WORDS Centipedes; checklist; new record; Cameroon.

Received 22.05.2020; accepted 01.07.2020; published online 13.08.2020

\section{INTRODUCTION}

Centipedes (Chilopoda) encompass more than 3300 species, divided into five extant orders, and are known to be from all continents, except Antarctica (Bonato et al., 2016). They are found to occur on the litter, under decaying woods, inside termite mounds as well as anthropogenic areas. Centipedes are important predators in most soil communities throughout the world. Their body is elongated, in a worm-like shape, and often flattened. The first pair of trunk appendages are developed as stinging fangs (also called forcipules), which are used to catch prey and for defence (Bonato \& Minelli, 2004; Guizze et al., 2016). Centipedes mainly prey on other arthropods, but they are also able to capture earthworms and small vertebrates (Lewis, 1981; Edgecombe \& Giribet, 2007; Noronha et al., 2015)

The Centipede fauna of Cameroon, one of the largest countries in central Africa, is still littleknown, while the only monograph devoted to the subject (Porat, 1894) is hopelessly outdated. De- spite being common, conspicuous, diverse, often easy-to-collect, Chilopoda have received little attention in all areas of zoological research in this country. Many available reports from Cameroon Myriapoda fauna have focused on systematic, taxonomy and ecology of millipedes. This neglect has mainly been due to the lack of experts in this new research domain in Cameroon.

The present checklist provides a review of the centipedes from old reports and recent field data with updated taxonomic names and new distribution records. This checklist is to be regarded as an invitation to further studies on the still poorlyknown arthropod class Chilopoda.

\section{MATERIAL AND METHODS}

The classification for Chilopoda follows Minelli (2011). All scientific names were updated according to the recent centipede taxonomic website: http://www.chilobase.biologia.unipd.it (Bonato et al., 2016). Families within order, genera within 
families, and species within genera are listed alphabetically. In the catalogue, the valid name of genus and species are given in bold and original descriptions are listed. Generic synonymy and/or type species provenances is listed under the valid name. In species synonymy, only references to the fauna of Cameroon were cited after original description. The cited references were followed by codification distinguishing publications with (1) taxonomic description and (2) merely listing or mention. Records from Cameroon are given with references. New records from Cameroon are given with number of specimens (male and/or female), place and georeference coordinates, date, and collector's name. The known general distribution of the species is given. Notes in terms of taxonomic status or misidentifications are given (if applicable).

\section{RESULTS}

\section{CHECKLIST}

Classis CHILOPODA Latreille, 1817 Ordo LITHOBIOMORPHA Pocock, 1910

Familia HENICOPIDAE Pocock, 1901

Genus Lamyctes Meinert, 1868

TyPE-SPECIES. Lamyctes fulvicornis Meinert, 1868 - by original designation.

SynONYMS. Lamyctinus Silvestri, 1909; Remylamcystes Attems, 1951

Lamyctes (Metalamyctes) africanus (Porat, 1871)

Henicops africana Porat, 1871: 1140 (1); 1893: 5 (2); 1894: 10 (2).

Records from Cameroon. Bonge (Porat, 1871).

Distribution. Cameroon, Madagascar, Senegal, Hawalian and Juan Fernandez islands (Pocock, 1888; Attems, 1903; Zapparoli \& Shelley, 2000).

Ordo SCOLOPENDROMORPHA Pocock, 1910

Familia CRYPTOPIDAE Kohlrausch, 1881

Genus Cryptops Leach, 1814

TYPE-SPECIES. Scolopendra hortensis Donovan, 1810 - by monotypy.
Cryptops (Cryptops) hortensis (Donovan, 1810)

Cryptops hortensis Donovan, 1810: 23(1); Porat, 1894: 16 (2).

RECORDS FRom CAMEROON. Bonge (Porat, 1894).

New RECORDs. 2 males, Campo Ma'an National Park, $2^{\circ} 10^{\prime} \mathrm{N}, 9^{\circ} 50^{\prime} \mathrm{E}, 30 . \mathrm{IX} .2015$, coll. Mbenoun \& Nzoko; 1 female, same location, $2^{\circ} 10^{\prime} \mathrm{N}$, $9^{\circ} 50^{\prime} \mathrm{E}, 31$.VIII. 2015, coll. Mbenoun \& Nzoko; 1

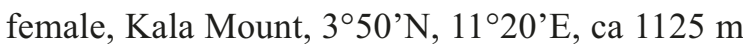
a.s.1., 29.I.2017, coll. Mbenoun \& Makon (see Mbenoun et al., 2017: Mbenoun \& Makon, 2019).

Distribution. Widespread in temperate and tropical regions throughout the world, often introduced (Sseliwanoff, 1884; Porat, 1894; Attems, 1903; Verhoeff, 1943; Chamberlin, 1944; Goux, 1950; Kock, 1863).

Note. This species occurs mostly in temperate climate and unlikely to survive in environmental conditions different from those in its native range. Its presence in Africa required critical evaluation. Introduced specimens may occur in city gardens and similar habitats to those found in its native range.

Cryptops (Trigonocryptops) gigas Kraepelin, 1903

Cryptops (Trigonocryptops) gigas Kraepelin, 1903: 40 (1).

ReCordS From CAMERoOn. Cameroon, without further details (Kraepelin, 1903).

Distribution. Only known from Cameroon.

Familia SCOLOPOCRYPTOPIDAE Pocock, 1896 Genus Scolopocryptops Newport, 1844

TYPE-SPECIE. Scolopocryptops melanostoma Newport, 1845.

SyNONYMS. Anethops Chamberlin, 1902; Dinocryptops Crabill, 1953; Otocryptops Haase, 1887.

Scolopocryptops ferrugineus (Linnaeus, 1767) Scolopocryptops ferrugineus Linnaeus, 1767: 1063 (1). 
Otocryptops ferruginea Porat, 1893: 12 (2); 1894: $16(2)$.

RECORDS From CAMEROON. Bonge (Porat, 1893).

New RECORDS. 1 male, Kala Mount, $3^{\circ} 50^{\prime} \mathrm{N}$, $11^{\circ} 20^{\prime} \mathrm{E}$, ca $1125 \mathrm{~m}$ a.s.1., 29.IX.2017, coll. Mbenoun \& Makon (Mbenoun \& Makon, 2019).

Distribution. West Africa, Central America, Caribbean (Linnaeus, 1797; Gervais, 1847; Marshall, 1878; Pocock, 1888; Humbert \& Saussure, 1869).

Note. The synonymy between Otocryptops ferruginea and Scolocryptops ferrugineus is also given by many authors, but both type materials require further revision.

Familia SCOLOPENDRIDAE Newport, 1844 Genus Alipes Imhoff, 1854

TYPE-SPECIES. Alipes multicostis Imhoff, 1854, by monotypy.

SYNONYMS. Eucorybas Gerstaecker, 1854.

Alipes multicostis Imhoff, 1854

Alipes multicostis Imhoff, 1854: 120 (1); Porat, 1894: 15 (2).

Records from CAmeroon. Kitta, Bonge (Porat, 1894).

Distribution. Cameroon, Guinea, Ivory Coast, Ghana (Porat, 1894; Imhoff, 1853; Demange, 1963).

Genus Ethmostigmus Pocock, 1898

TyPE SPECIES. Scolopendra trigonepodus Leach, 1816 - by subsequent designation.

SynONYMS. Dacetum, Kock, 1847; Heterostoma Newport, 1844.

Ethmostigmus trigonepodus (Leach, 1817) Ethmostigmus trigonepodus Leach, 1817: 36 (1). Heterostoma trigonopodum Porat, 1894: 15 (2).

ReCordS From CAMEROON. Bibundi (Porat, 1894).

Distribution. Cameroon, Algeria, Tanzania, Angola, Congo, Ethiopia, Malawi, Sudan, South
Africa; Tanzania (Koch, 1847; Porat, 1894; Verhoeff, 1941).

Genus Scolopendra Linnaeus, 1758

TYPE SPECIES. Scolopendra morsitans Linnaeus, 1758.

SYNONYMS. Scolopendra (Calcaria) Porat, 1876; Rhombocephalus Newport, 1844; Trachycormocephalus Kraepelin, 1903; Rhadinoscytalis Attems, 1926; Kanparka Waldock \& Edgecombe, 2012.

Scolopendra morsitans Linnaeus, 1758

Scolopendra morsitans Linnaeus, 1758: 638 (1).

Scolopendra morsitans Porat, 1894: 11 (2).

ReCords from CAMERoOn. Kitta, Ekundu, Bibundi (Porat, 1894).

New RECORds. 1 male, Mahonda, $4^{\circ} 15^{\prime} \mathrm{N}$; 1048'E, 14.XI.2013, coll. Mbenoun.

DisTRIBUTION. This well-known species is found in almost all tropical and subtropical countries (Brandt, 1841; Newport, 1844; Porat, 1894; Khanna, 1977).

Note. Frequenty introduced.

Scolopendra valida Lucas, 1840

Scolopendra valida Lucas, 1840: 49 (1).

ReCords From CAMERoOn. Cameroon, without further details (Lucas, 1840).

Distribution. Cameroon, Sudan, Somalia, Canary Islands, Iran, India, Kuwait (Lucas, 1840, Lewis, 2010; Reeves, 2016).

Note. This species has been previously omitted in Porat's checklist (1894).

Genus Otostigmus Porat, 1876

TyPE SPECIES. Otostigmus carinatus Porat, 1876 - by subsequent designation

SyNONYM. Branchiotrema Kohlrausch, 1878

Otostigmus (Otostigigmus) inermipes Porat, 1893

Otostigmus inermipes Porat, 1893 :11(1); 1894 :14

(2). 
Records from CAMERoon. Bibundi, Mapanja, Bonge (Porat, 1893).

Distribution. Only known from Cameroon.

Otostigmus (Parotostigmus) cuneiventris Porat, 1893

Otostigmus cuneiventris Porat, 1893: 10 (1) ; 1894: 14 (2).

Records from Cameroon. Bibundi, Mapanja, Bonge (Porat, 1893).

Distribution. Cameroon, Tanzania (Porat, 1893; Attems, 1930).

Ordo GEOPHILOMORPHA Pocock, 1895

Familia GEOPHILIDAE Leach, 1815

Genus RIBAUTIA Brölemann, 1909

TyPe SPECIES. Ribautia bouvieri Brölemann, 1909 - by monotypy.

Synonyms. Schizoribautia Brölemann, 1912; Polygonarea (Nearia) Chamberlin, 1955.

Ribautia unguiculata (Porat, 1894)

Geophilus unguiculatus Porat, 1894: 23 (1).

Records from Cameroon. Patras (Porat, 1894).

Distribution. Cameroon, Benin, Togo, Democratic Republic of Congo (Porat, 1894; Cook, 1896; Brolemann, 1926).

Genus Schizotaenia Cook, 1896

TyPE SPECIES. Schizotaenia prognatha Cook, 1896 - by subsequent designation.

Schizotaenia aequalis (Porat, 1894)

Geophilus aequalis Porat, 1894: 23 (1).

ReCORdS From CAMEROON. Cameroon, without further details (Porat, 1894).

Distribution. Only known from Cameroon.

Schizotaenia porosa (Porat, 1894)

Geophilus porosus Porat, 1894: 22 (1).

Records from Cameroon. Ekundu; Bonge (Porat, 1894).
DisTRIBUTION. Only known from Cameroon.

Schizotaenia quadrisulcata (Porat, 1894)

Geophilus quadrisulcatus Porat, 1894: 23 (1).

RECORDS FRom CAMEROON. Bonge (Porat, 1894).

Distribution. Only known from Cameroon.

Genus Tretechthus Cook, 1896

TyPESPECIES. Geophilus uliginosus Porat, 1894

- by monotypy.

Tretechthus uliginosus (Porat, 1894)

Geophilus uliginosus Porat, 1894: 24 (1)

Records from CAMERoOn. Cameroon, without further details (Porat, 1894).

Distribution. Only know from Cameroon.

Familia MECISTOCEPHALIDAE Bollman, 1893 Genus Mecistocephalus Newport, 1843

TYPE SPECIES. Mecistocephalus punctifrons Newport, 1843 - by subsequent designation.

SyNONYMS. Lamnonyx Cook, 1896; Megethmus Cook, 1896; Pauroptyx Chamberlin, 1920; Brachyptyx Chamberlin, 1920; Dasyptyx Chamberlin, 1920; Ectoptyx Chamberlin, 1920; Formosocephalus Verhoeff, 1937; Fusichila Chamberlin, 1953.

Mecistocephalus punctifrons Newport, 1843

Mecistocephalus punctifrons Newport, 1843: 179 (1); Porat, 1894: 20 (2).

New RECORdS. 1 male, Kala Mount, $3^{\circ} 50^{\prime} \mathrm{N}$, $11^{\circ} 20^{\prime} \mathrm{E}$, ca $1125 \mathrm{~m}$ a.s.1., 06.I. 2017, coll. Mbenoun \& Makon; 1 female, same locality, ca $820 \mathrm{~m}$ a.s.1, 24.II.2017, coll. Mbenoun \& Makon; 1 male, same locality, ca $1125 \mathrm{~m}$ a.s.1, 19.IV.2017, coll. Mbenoun \& Makon; 1 female, same locality, 25.XI.2016, coll. Mbenoun \& Makon; 1 male, same locality, 08.IV.2017, coll. Mbenoun \& Makon; 2 males, same locality, 29.IV.2017, coll. Makon; 2 females, same locality, 06.I.2017, coll. Mbenoun \& Makon (see Mbenoun et al., 2017: Mbenoun \& Makon, 2019). 
Distribution. India (Bonato \& Minelli, 2004).

Notes. According to Bonato \& Minelli (2004), M. punctifrons is exclusive in India and records from Cameroon have been most probably based on misidentifications. Therefore, critical evaluation is needed. Most probably the populations inhabiting Africa are likely to be $M$. togensis.

Mecistocephalus togensis (Cook, 1896) Lamnonyx togensis Cook, 1896: 39 (1).

Records from CAMERoon. Ototomo (Edgecombe et al., 2010).

Distribution. Cameroon, Togo (Cook, 1896; Edgecombe et al., 2010).

Notes. The synonymy with Mecistocephalus insularis, M. guildingii, M. leonensis has been proposed by some authors.

Familia ORYIDAE Cook, 1896

Genus Orphnaeus Meinert, 1870

TyPE SPECIES. Orphnaeus lividus Meinert, 1870 - by subsequent designation.

SynONYMS. Andenophilus Verhoeff, 1941; Azygethus Chamberlin, 1920.

Orphnaeus brasilianus (Humbert et Saussure, 1870)

Orphnaeus lineatus Newport, 1845: 437(1); Porat, 1894: 19 (2).

ReCords From CAMEROON. Cameroon, without further details (Porat, 1894).

Distribution. Cameroon, Madagascar, Hunduras, Rio Grande do Norte (Brazil), Africa (Newport, 1844; Porat, 1894; Attems, 1903; Chamberlin, 1914).

Note. The old record $O$. lineatus by Porat (1894) is probably $O$. brasilianus. Therefore, critical evaluation is needed.

Orphnaeus brevilabiatus (Newport, 1845) Geophilus brevilabiatus Newport, 1845: 436 (1). Orphnaeus brevilabiatus Porat, 1894: 19 (2); 1893: 14 (2).
Records from Cameroon. Mapanja (Porat, 1894).

DISTRIBUTION. This species is widely distributed in the tropical and subtropical parts of both Eastern and Western Hemispheres.

Genus Pentorya Cook, 1896

Type SPECIES. Pentorya afra Cook, 1896 - by original designation

Pentorya afra Cook, 1896

Pentorya afra Cook, 1896: 34 (1).

Orphnaeus (Aspidopleres) fusatus Porat 1894: 18

Records from CAmeroon. Ekundu (Porat, 1894).

Distribution. Cameroon.

Familia SCHENDYLIDAE Cook, 1896

Genus Ballophilus Cook, 1896

TYPE SPECIES. Ballophilus clavicornis Cook, 1896 - by subsequent designation.

Ballophilus maculosus (Porat, 1894) Geophilus maculosus Porat, 1894: 25 (1).

Records from Cameroon. Bonge (Porat, 1894).

Distribution. Only known from Cameroon.

Genus Ctenophilus Cook, 1896

TYPE-SPECIES. Ctenophilus africanus Cook, 1896 - by subsequent designation.

SynONYM. Pleuroschendyla Brölemann et Ribaut, 1911.

Ctenophilus edentulus (Porat, 1894)

Geophilus edentulus Porat, 1894: 24 (1).

Records from CAmeroon. Bonge (Porat, 1894).

Distribution. Cameroon. 
Ordo SCUTIGEROMORPHA Pocock, 1895 Familia PSELLIODIDAE Chamberlin, 1955 Genus Sphendononema Verhoeff, 1904

TYPE SPECIES. Sphendononema camerunense Verhoeff, 1904 - by original designation.

SYNONYMS. Pselliophora Verhoeff, 1904; Pselliodes Chamberlin, 1921; Brasilophora Bücherl, 1939; Lassothereua Verhoeff, 1944.

Sphendononema rugosa (Newport, 1844) Sphendononema rugosa Newport, 1844: 95 (1)

RECORDS FROM CAMEROON. Cameroon, without further details (Verhoeff, 1904, 1905).
Distribution. Cameroon, Angola, Gabon, Guinea, Kenya (Verhoff, 1904, 1905).

\section{CONCLUSIONS}

The chilopod fauna of Cameroon encompasses 23 species and subspecies, nine families, and four orders including Lithobimorpha (one species), Scutigeromorpha (one species), Scolopendromorpha (9 species) and Geophilomorpha (12 species) (Table 1). Nine species (39\%) are so far only known from Cameroon: Pentorya afra Cook, 1896; Ctenophilus edentulus (Porat, 1894); Tretechthus uliginosus (Porat, 1894); Schizotaenia quadrisulcatus (Porat,

\begin{tabular}{|c|c|c|}
\hline Order & Family & Species \\
\hline LITHOBIOMORPHA & HENICOPIDAE & Lamyctes (Metalamyctes) africanus (Porat, 1871) \\
\hline SCUTIGEROMORPHA & PSELLIODIDAE & Sphendononema rugosa (Newport,1844) \\
\hline \multirow[t]{9}{*}{ SCOLOPENDROMORPHA } & CRYPTOPIDAE & Cryptops (Cryptops) hortensis (Donovan, 1810) $\neq$ \\
\hline & & Cryptops (Trigonocryptops) gigas Kraepelin, 1903* \\
\hline & SCOLOPOCRYPTOPIDAE & Scolopocryptops ferrugineus (Linnaeus, 1767) $\neq$ \\
\hline & SCOLOPENDRIDAE & Alipes multicostis Imhoff, 1854 \\
\hline & & Ethmostigmus trigonepodus (Leach, 1817) \\
\hline & & Otostigmus (Otostigmus) cuneiventris Porat, 1893 \\
\hline & & Otostigmus (Parotostigmus) inermipes Porat, 1893* \\
\hline & & Scolopendra morsitans Linnaeus, $1758 \neq$ \\
\hline & & Scolopendra valida Lucas, 1840 \\
\hline \multirow[t]{12}{*}{ GEOPHILOMORPHA } & SCHENDYLIDAE & Ballophilus maculosus (Porat, 1894) * \\
\hline & & Ctenophilus edentulus (Porat, 1894) * \\
\hline & GEOPHILIDAE & Ribautia unguicula (Porat, 1894) \\
\hline & & Schizotaenia aequalis (Porat, 1894) * \\
\hline & & Schizotaenia porosa (Porat, 1894) * \\
\hline & & Schizotaenia quadrisulcatus (Porat, 1894) * \\
\hline & & Tretechthus uliginosus (Porat, 1894) * \\
\hline & MECISTOCEPHALIDAE & Mecistocephalus punctifrons Newport, $1843 \neq$ \\
\hline & & Mecistocephalus togensis (Cook, 1896) \\
\hline & ORYIDAE & Orphnaeus brasilianus (Humbert et Saussure, 1870) \\
\hline & & Orphnaeus brevilabiatus (Newport, 1845) \\
\hline & & Pentorya afra Cook, 1896 * \\
\hline
\end{tabular}

Table 1. Overview of the centipede fauna of Cameroon. Classification after Minelli et al. (2012). * indicates endemic species; $\neq$ indicates new records. 
1894); Schizotaenia porosa (Porat, 1894); Schizotaenia aequalis (Porat, 1894); Ballophilus maculosus (Porat, 1894); Otostigmus (Parotostigmus) inermipes Porat, 1893; and Cryptops (Trigonocryptops) gigas Kraepelin, 1903. Six species (26\%) are known from the surrounding African countries: Ribautia unguicula (Porat, 1894); Otostigmus (Otostigmus) cuneiventris Porat, 1893; Ethmostigmus trigonepodus (Leach, 1817); Alipes multicostis Imhoff, 1854; Mecistocephalus togensis (Cook, 1896), and Sphendononema rugosa (Newport, 1844).

The overall number of centipede species (23) recorded from the historical reports and recent field data in Cameroon is greater than the number recorded to the fauna of Gabon (15 species) (Demange, 1968). Although few diversity studies have been conducted, centipedes seem to be more diverse and abundant in Cameroon (Mbenoun \& Makon, 2019). Due to the lack of relevant literature and expertise of this taxonomic group, many unidentified or doubtful species have been excluded from the current list. The identification of these morphospecies may probably increase the number of centipedes present in the country. Most of data published in this catalogue were collected mainly by Porat (1894) for decades with a few under-staffed expeditions in the south-western region of Cameroon. This region is located in southern Cameroon rainforest and characterized by lowland evergreen and montane forests. A great majority of species found by Porat (1894) are likely to be endemic in Cameroon. The old data recorded from literature and those described by Porat therefore need careful revision. Accordingly, surveys should be performed in the other geographic regions within southern Cameroon rainforest so as to document their diversity and thus contribute to the knowledge of this poorly known Myriapoda class in the country.

\section{ACKNOWLEDGEMENTS}

Thanks are due to Alessandro Minelli and Lucio Bonato (Dipartimento di Biologia, Università degli Studi di Padova, Italia) for their valuable comments on the final draft of this work. We are grateful to S.I. Golovatch (Institute for Problems of Ecology and Evolution, Russian Academy of Sciences, Moscow), for commenting on the early version of the manuscript.

\section{REFERENCES}

Attems C., 1903. Synopsis der Geophiliden. Zoologische Jahrbücher, Abteilung für Systematik, 18: 155302.

Bonato L. \& Minelli A., 2004. The centipede genus Mecistocephalus Newport 1843 in the Indian Peninsula (Chilopoda Geophilomorpha Mecistocephalidae). Tropical Zoology, 17: 15-63. https://doi.org/10. 1080/ 03946975.2004.10531198

Brandt J.F., 1841. Note supplémentaire sur quelques espèces du genre de scolopendre suivie de la description de deux espèces nouvelles et d'un essai d'une subdivision de ce genre en deux sous-genres. Bulletin Scientifique, Académie Imperiale des Sciences de Saint Pétersbourg, 8: 21-25.

Brolemann H.W., 1926. Myriapodes recueillis en Afrique Occidentale Francaise par M. l'Administrateur en chef L. Duboscq - Archives de Zoologie Expérimentale et Générale, 65: 1-159.

Bonato L., Chagas Junior A., Edgecombe G.D., Lewis J.G.E., Minelli A., Pereira L.A., Shelley R.M., Stoev P. \& Zapparoli M., 2016. ChiloBase 2.0. A World Catalogue of Centipedes (Chilopoda). Available at https://chilobase.biologia.unipd.it. (Accessed on 22th may, 2020)

Chamberlin R.V., 1944. Chilopods in the collections of Field Museum of Natural History - Field Museum Natural History Publications. Zoological Series, 28: 175-216. https://doi.org/10.5962/bhl.title.3098

Cook O.F., 1896. Geophiloidea from Liberia and Togo. Brandtia, 35-40.

Demange J. M., 1968. Myriapodes chilopodes du Gabon. Extrait de la revue "'Biologia gabonica", 3: 281-294.

Demange J.M., 1963. Chilopoda. La réserve naturelle integrale du Mont Nimba. Mémoires de l'Institut Francaise d'Afrique Noire, 5: 41-118.

Edgecombe G.D. \& Giribet G., 2007. Evolutionary biology of centipedes (Myriapoda: Chilopoda). Annual Review of Entomology, 52: 151-170. https://doi.org/ 10.1146/annurev.ento.52.110405.091326

Edgecombe G.D., Bonato L. \& Girbert G., 2010. Brooding in Mecistocephalus togoensis (geophilomorpha: Placodesmata) and the evolution of parental care in centipedes (Chilopoda). International journal of Myriapodology, 3: 139-144.

Gervais P., 1847. Myriapodes. In: Walckenaer C.A. \& Gervais F.L.P. (Eds.), Histoire naturelle des Insectes Aptères, Tome 4, Paris: Librairie Encyclopédique de Roret, 210-623.

Goux L., 1950. Contribution à l'étude des myriapodes de la France. I. Description d'un Cryptops nouveau (Chilop. Cryptopsidae). Bulletin Mensuel de la Société Linnéenne de Lyon, 19: 195-199.

Guizze S.P.G., Knysak I., Barbaro K.C., Karam-Gemael 
M. \& Chagas-Junior A., 2016. Predatory behavior of three centipede species of the order Scolopendromorpha (Arthropoda: Myriapoda: Chilopoda). Zoologia, 33 e20160026. https:// doi.org/ 0.1590/S1984-4689 zool- 20160026

Humbert A. \& Saussure E. de (1869). Myriapoda nova Americana. Revue et Magasin de Zoologie Pure et Appliquée, 21: 149-159.

Khanna V., 1977. Studies on the centipede genus Trachycormocephalus (Myriapoda: Scolopendridae) from Rajasthan, India. Oriental Insects, 11: 151-156. https://doi.org/10.1080/00305316.1977.10432088

Koch C.L., 1847. System der Myriapoden. In: HerrichSchäffer L. (Ed.), Kritische Revision der Insectenfauna, Deutschlands: Pustet, Regensburg, 1-270.

Koch C.L., 1863. Die Myriapoden getreu nach der Natur abgebildet und beschhrieben. H. W. Schmidt, Halle, 246 pp. https://doi.org/10.5962/bhl.title.10639

Kraepelin., 1903. Revision der Scolopendriden. Mitteilungen aus dem Naturhistorischen Museum, Hamburg, 20: 1-276.

Lewis J.G.E., 2010. A key and annotated list of the Scolopendra species of the Old World with a reappraisal of Arthrorhabdus (Chilopoda: Scolopendromorpha: Scolopendridae). International Journal of Myriapodology, 3: 83-122. https://doi.org/10.1163/ 187525410X 12578602960380

Lewis J.G.E., 1981. The Biology of Centipedes. Cambridge, Cambridge University Press, $474 \mathrm{pp}$. https://doi.org/10.1017/CBO9780511565649

Marshall T.A., 1878. Arachnida and Myriopoda - Proceedings of the Entomological Society of London, 37: 36-39.

Mbenoun Masse P.S. \& Makon S.M., 2019. Effects of an altitudinal gradient on myriapod diversity and abundance on Mount Kala, central Cameroon. African Zoology 54: 215-223. https://doi.org/10.1080/1562 7020.2019.1677496

Minelli A., 2011. Anatomy, Biology, Taxonomy the Myriapoda. Treatise on Zoology, Volume 1, 523 pp.

Newport G., 1844. Monograph of the class Myriapoda order Chilopoda; with observations on the general arrangement of the Articulata. Transactions of the Lin- nean Society of London, 19: 265-302. https://doi. org/10.1111/j.1096-3642.1842.tb00368.x

Noronha J.C., BattiroLa L.D., Chagas-Junior A., Miranda R.M., Carpanedo R.S. \& Rodrigues D.J., 2015. Predation of bat (Molossus molossus: Molossidae) by the centipede Scolopendra viridicornis (Scolopendridae) in Southern Amazonia. Acta Amazonica, 45: 333336. https://doi.org/10.1590/1809-4392201404083

Pocock R.I., 1888. Contributions to our knowledge of the Myriopoda of Dominica - Annals and Magazine of Natural History, 2: 472-483. https:// doi.org/10.1080/ 00222938809487517

Porat C.O., 1893. Myriopoder fran Vest och Syd-Afrika." Bihang Till K. Svenska Vetenskaps-Akademiens Handlinga, 18: 1-51.

Porat C.O., 1894. Zur Myriopodenfauna Kameruns. Bihang Till K. Svenska Vetenskaps-Akademiens Handlingar, 20: 1-90.

Porat C.O., 1871. Myriopoda Africae australis in Museo Regio Holmiensi asservata recensuit. Pars I. Chilopoda - Öfversigt af Kongliga VetenskapsAkademiens Förhandlingar, 28: 1135-1167.

Reeves W.L., 2016. First national records for Scolopendra valida (Chilopoda: Scolopendromorpha: Scolopendridae) from Kuwait. Entomological news, 128: 151152. https://doi.org/10.3157/021. 126.0212

Sseliwanoff A.,1884. Materialii k isyceniu russkich tuisiacenogech (Myriapoda). Horae Societatis Entomologicae Rossicae, 18: 69-121.

Verhoeff C.W., 1904. Über Gattungen der Spinnensseln (Scutigeriden). Sitzungs-Beri-chte der Gesellschaft naturforschender Freunde zu Berlin, 1904: 245-285.

Verhoeff C.W., 1905. Über Scutigeriden. 5. Aufsatz - Zoologischer Anzeiger, 29: 73-119.

Verhoeff K.W., 1943. Neuer Beitrag zur Kenntnis der Chilopoden der Insel Ischia. Zoologischer Anzeiger, 142: 62-83.

Verhoeff K.W., 1941. Aliquid novi ex Africa. IV. Zoologischer Anzeiger, 135: 196-204.

Zapparoli M. \& Shelley R.M., 2000. The centipede order Lithobiomorpha in the Hawaiian islands (Chilopôda). I. The epigean fauna. Bishop Museum Occasional Papers, 63: 35-49. 